\title{
Genitourinary System Disorder
}

National Cancer Institute

\section{Source}

National Cancer Institute. Genitourinary System Disorder. NCI Thesaurus. Code C156660.

A non-neoplastic or neoplastic disorder that affects the genitourinary system. 